\title{
SIGNAL-TO-NOISE MEASURES FOR MAGNETIC RESONANCE IMAGERS
}

\author{
B.W. Murphy, P.L. Carson, J.H. Ellis, Y.T. Zhang, R.J. Hyde, and T.L. Chenevert \\ Department of Radiology, Ann Arbor Veterans Affairs Medical Center, Ann Arbor, MI 48105, USA, and \\ University of Michigan Medical Center, Ann Arbor, MI 48109-0553, USA
}

\begin{abstract}
The signal-to-noise ratio (SNR) in magnetic resonance imaging represents one of the system operating variables that must be determined both for evaluating the performance of different imaging protocols on a particular machine, and for monitoring machine performance as part of a routine quality control (QC) program. Utilizing a phantom and set of automated analysis programs currently under development, this study evaluated several ways of measuring image signal and noise and demonstrated the importance of utilizing measured voxel volumes as opposed to nominal volumes in the calculation of SNR. The NEMA proposed standard for SNR is compared with several other SNR measures and is recommended as the measure to be used in routine SNR reporting. The importance of utilizing other SNR measures in addition to the NEMA proposed standard for routine QC is discussed.
\end{abstract}

Keywords: MRI; Quality assurance; Signal-to-noise; Phantom; Image processing; Automation.

\section{INTRODUCTION}

There exists an extensive literature on the importance of determining the signal-to-noise ratio (SNR) in magnetic resonance (MR) images as well as in techniques for performing the measurement. All of these methods address the fundamental dependence of SNR on voxel volume but tend to make the assumption that the volume is constant both temporally and spatially. This is achieved by either fixing the imaging protocol or normalizing by a particular protocol's nominal voxel volume, or both. This simplification is made because the determination of actual voxel volume over the whole imaging volume is impractical, requiring a specialized phantom as well as the time consuming manual measurement of the resulting image by either service personnel or medical technologists. Added to this is the need to perform this measurement with every determination of SNR to account for temporal changes in the voxel volume.

The noise in an image may be determined in several ways. It can be measured directly from a large uniform, signal region as the standard deviation of pixel values in that region. ${ }^{1}$ Another approach which may be employed on magnitude reconstructed images is that of estimating the noise from the signal amplitude value of nonsignal regions. ${ }^{1}$ Alternatively, the noise can be determined by acquiring two images of the same object, subtracting one from the other and finding the standard deviation of the difference image. This last technique is the one currently advocated by National Electrical Manufacturers Association (NEMA). ${ }^{2}$ It has an advantage over the first two measures of noise in that, when properly employed, it is relatively insensitive to structured noise, for example, noise due to phenomena such as ghosting, ringing across edge boundaries due to finite sampling, and so on.

\section{METHOD}

At our institution, we have developed a parallel rod test object (PRoTO) and a set of computer implemented analysis programs. An image of the cross section of the PRoTO has a checker board appearance consisting of signal and nonsignal blocks. The analysis programs automatically extract many machine performance variables from a set of images of a single scan of the phantom and utilize them in routine quality control (QC) procedures being developed. ${ }^{3,4}$ One of the advantages that this phantom and set of analysis routines provides is the easy determination of signal, voxel volume ( $x, y$ pixel sizes and slice thickness), and various measures of noise at many locations within a slice (for each slice) of a single scan. This makes the phantom and programs ideal for exploring the significance of variables incorporated in the SNR. The PRoTO was
RECEIVED 6/24/92; ACCEPTED 11/12/92.

Address correspondence to Dr. P. L. Carson, University of Michigan Medical Center, 1500 E. Medical Center Drive, Ann Arbor, MI 48109-0553. 
routinely imaged on a $1.5 \mathrm{~T}$ MRI unit (Signa, General Electric Medical Systems, Milwaukee, WI) using a fixed imaging protocol (SE500/30, $40 \mathrm{~cm}$ field of view, $256 \times 256$ image, $2 \mathrm{NEX}$, interleaved slices, no interslice gap, that is, slice thickness = slice separation).

\section{RESULTS AND DISCUSSION}

Figure 1 represents a simple measure of signal intensity from the signal blocks of the PRoTO which have not been corrected for voxel volume. While one should expect a slight roll-off in signal in the outer planes due to filtering, one certainly does not expect the sawtoothed regularity observed. Compare this to Fig. 2 where the signal per measured voxel volume has been plotted. This corrected data does have a reasonable shape. The saw-tooth pattern of pixel signal intensities is caused by a variation in voxel volume which in turn is due to variations in slice thickness (Fig. 3). This variation in slice thickness was confirmed using a standard phantom designed for such purposes. The saw toothed pattern in the slice thickness is probably due to scanning the phantom using interleaving with no gap since this causes overlap of slice excitation. Such spatial variation in slice thickness leads us to conclude that not only should signal-to-noise values be reported with respect to voxel volume, but that the volume used in the calculations should be a measured volume. If the spatial variation in slice thickness were known to remain temporally constant then the determination of the voxel volume need only be done at acceptance of the MRI unit. However, the slice thickness and pixel size have been found to vary with time. Therefore, it becomes necessary for highest accuracy to quote the SNR relative to the voxel volume determined at the same time as the data for the SNR.

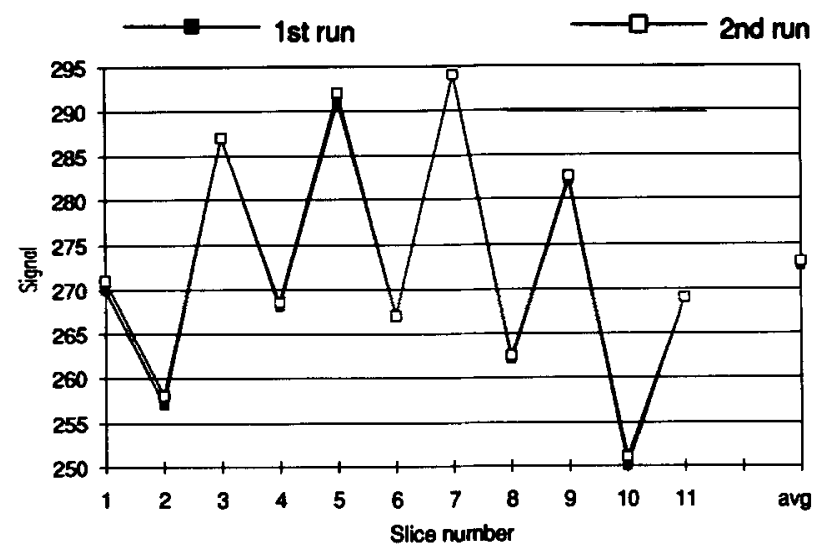

Fig. 1. Raw signal from the signal blocks of two consecutive scans of the PRoTO.

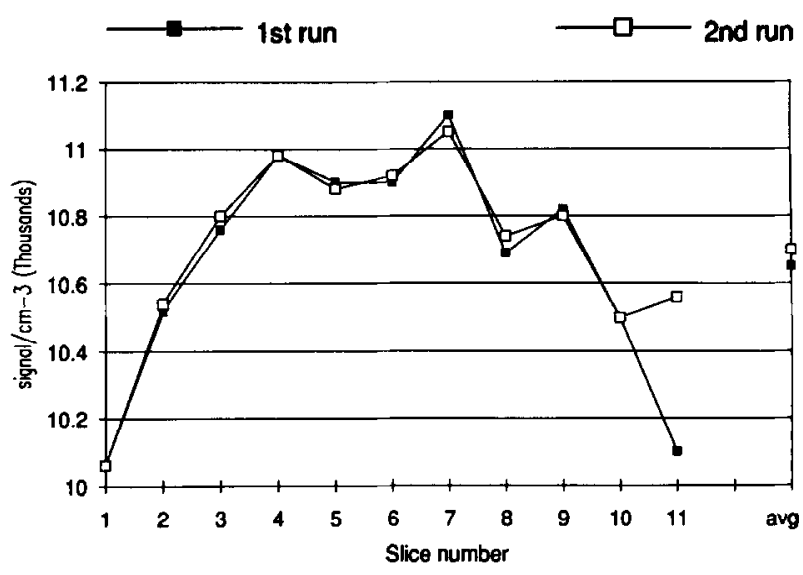

Fig. 2. Volume (measured) corrected signal for two consecutive scans of the PRoTO.

In Fig. 4, various measures of noise are plotted for 11 planes taken from two consecutive scans of our PRoTO phantom. It was necessary to take two scans in this way in order to generate the data required by the difference image method of computing image noise. The noise measures determined from either the mean signal amplitude from the nonsignal blocks or the standard deviation in either the signal amplitude of the signal or nonsignal areas reproduce well from the first scan to the second. However, the large variation in these measures across the slices, in particular the noise calculated from the signal amplitude from the nonsignal region, demonstrates that they are not appropriate for precise noise determination with the PRoTO phantom. The observed variation is due at least in part to ringing at the signal/nonsignal edge transition regions as a result of finite sampling of the PRoTO block edges. This ringing increases the average signal value in nonsignal regions as well as the standard deviation in both signal and nonsignal regions.

Contrast the single scan noise measures, discussed above, with the noise measures determined from the difference images. The latter method is reproducibly uniform across planes as well as yielding a consistent value for both signal and nonsignal regions of the PRoTO phantom. Hence, to evaluate accurately the noise level as a variable free of structured noise in an MR image, the technique recommended by NEMA is the correct procedure to utilize. Note that this latter finding of consistency between signal and nonsignal regions is not expected to hold in general. This is due to the fact that the estimate of noise from the nonsignal regions in images where the background is zero should be greater than the true noise by approximately $25 \%$, i.e., $(\pi / 2)^{1 / 2}$. $^{5}$ 

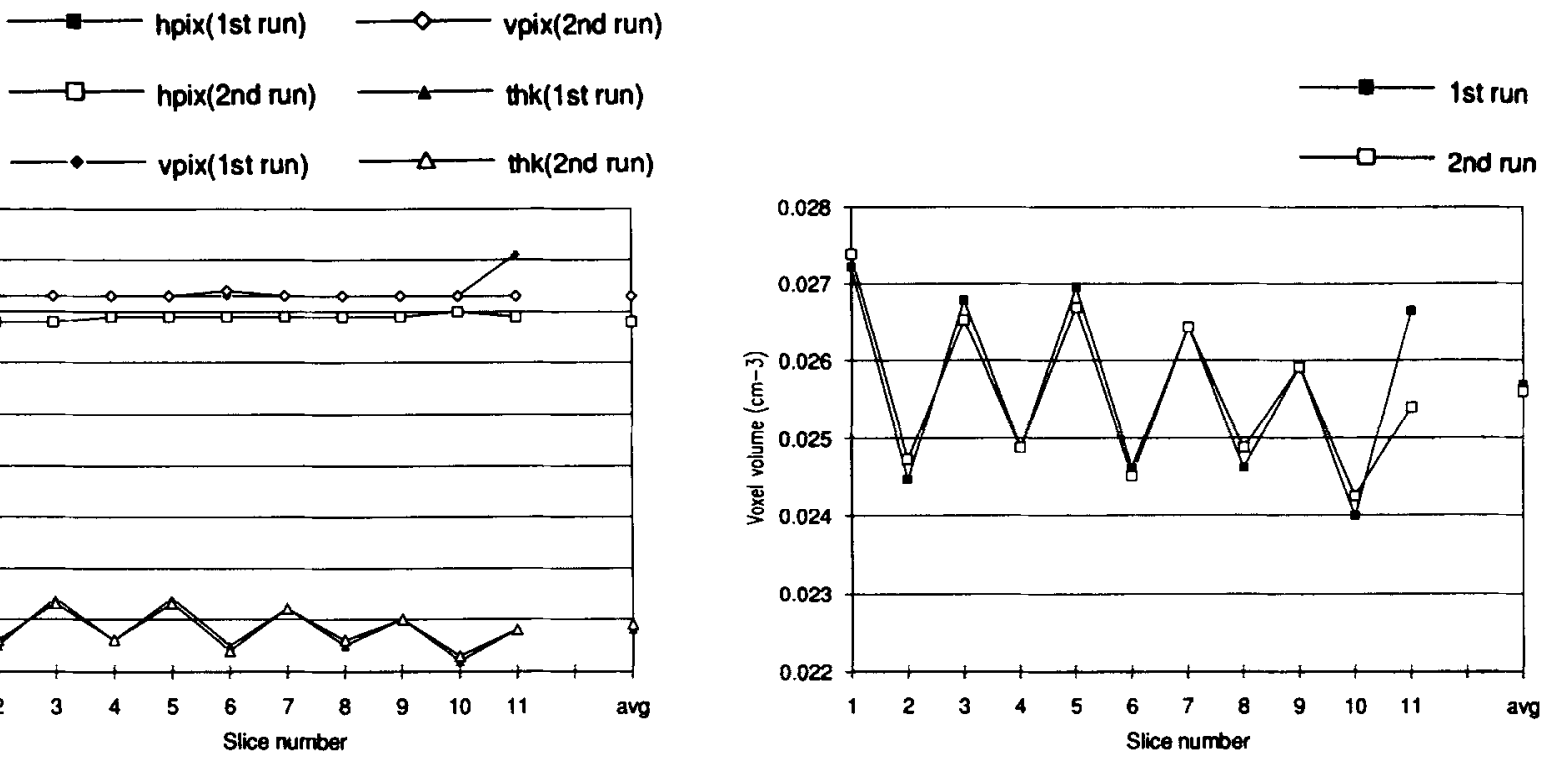

(A)

(B)

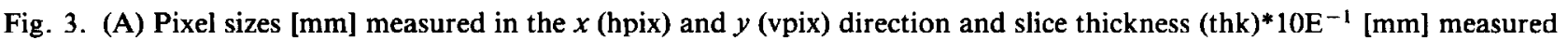
in the $z$ direction. (B) Measured voxel volume $\left[\mathrm{mm}^{-3}\right]$ (hpix*vpix*thk) for two consecutive scans of the PRoTO. It is the slice thickness that causes the variation in voxel volume.

Eliminating structure noise from noise measurements may result in masking out just the kind of information one would want to use for QC purposes. For example, monitoring the ratio of signal-block signal to either standard deviation of signal block signal or nonsignal block signal levels gives us information about the amount of ghosting occurring in the image. Since, for a given imaging protocol, the structured noise in the
PRoTO remains relatively constant, a degradation in machine performance which resulted in increased ghosting would be detected by one of these measures but not by the difference measure.

One technique for determining such structure noise may be to measure the noise at two locations within the scanner's sensitive region, yet outside the phantom, along both the phase encoding and read out directions.

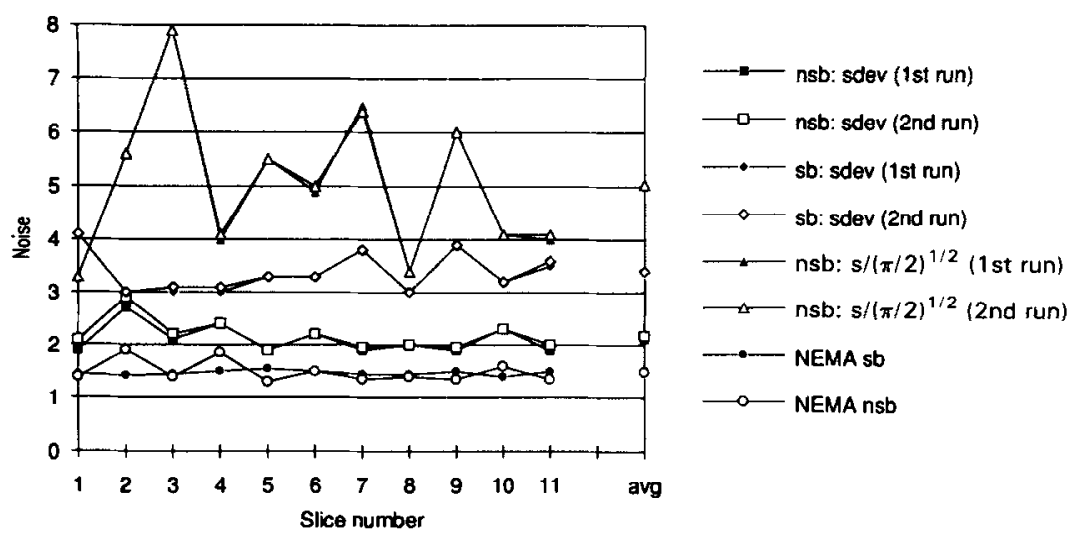

Fig. 4. Single slice noise measures compared to NEMA difference image noise measures generated using the PRoTO and analysis routines. PRoTO gives a checker board image consisting of signal and nonsignal areas. The single slice noise measures are derived from the signal amplitude (s) or standard deviation (sdev; $n-1$ weighting) of the signal amplitude from either the signal (sb) or nonsignal block (nsb) locations within the PRoTO. 
As most ghosting appears along the phase encoding direction, it may be possible to find a simple, linear scale factor that relates noise in the read out direction and noise determined from difference images. If such a factor can be shown to remain stable over time, then its ratio to the noise determined along the phase-encoding direction may yield another measure which is useful for monitoring ghosting in an image.

\section{CONCLUSIONS}

When considering signal and noise one must have a clear understanding of what is being measured and what information is contained in that measurement. Signal values reported should be reported as signal per voxel volume, and the voxel volume used should be a measured quantity. Failure to incorporate measured voxel volumes has been demonstrated to result in signal variation artifacts which make it impossible to compare any measures utilizing these signal values, either across MR scanners or even of one MR scanner at different times. Although measured voxel volume in each slice is not determined routinely, such information is easily acquired if an appropriate phantom, such as the PRoTO phantom, is employed.

After one has obtained a reliable measure of signal, understanding the various sources of noise, how they may be measured and the type of information they contain, determines how the reported SNR values can be interpreted. Routinely, one should report SNR determined from the difference images proposed by the NEMA standard. This measure has been demonstrated to be the most stable of the various noise measures dis- cussed when used with the PRoTO. For quality control, one should go beyond this single measure of SNR to include measures which are particularly sensitive to MR image artifacts such as ghosting, edge ringing, and other structure noise, as changes in any of these indicate underlying changes in MR scanner performance.

Acknowledgment - This work has been supported in part by the Veterans Affairs Merit Review Grant "Automated Analysis of Magnetic Resonance Imaging System Performance."

\section{REFERENCES}

1. Kaufman, L.; Kramer, D.M.; Crooks, L.E.; Ortendahl, D.A. Measuring signal-to-noise ratios in MR imaging. $R a$ diology 173:265-267; 1989.

2. Sano, R.M. NEMA standards: Performance standards for clinical magnetic resonance systems. In: R.L. Dixon (E.d). MRI Acceptance Testing and Quality Control-The Role of the Clinical Medical Physicist. Madison, WI: Medical Physics Publishing Company; 1988: pp. 185-189.

3. Covel, M.M.; Hearshen, D.O.; Carson, P.L.; Chenevert, T.L.; Shreve, P.; Aisen, A.M.; Bookstein, F.L.; Murphy, B.W.; Martel, W. Automated analysis of multiple performance characteristics in MRI systems. Med. Phys. 13(6): 815-823; 1986.

4. Carson, P.L.; Ellis, J.H.; Murphy, B.; Zhang, Y.; Liu, G. Automated analysis of magnetic resonance systems. In: R.L. Dixon (Ed). MRI Acceptance Testing and Quality Control - The Role of the Clinical Medical Physicist. Madison, WI: Medical Physics Publishing Company; 1988: pp. 151-173.

5. Henklemen, R.M. Measurement of signal intensities in the presence of noise in MR images. Med. Phys. 12(2):232233; 1985. 